\section{Defining normal heart and respiratory rates in children}

\author{
Roger C Parslow
}

In 2008, the Confidential Enquiry into Maternal and Child Health report, Why Children Die: A Pilot Study 2006, highlighted the need for health professionals to be able to identify a sick child and recommended greater use and awareness of validated scoring systems to identify acutely ill children. They made this recommendation based on their findings that a quarter of child deaths were preventable and "a major factor [relating to these deaths] was shortcomings in the recognition and management of the acutely ill child". ${ }^{1}$

The key word used here is 'validated': there are a variety of paediatric early warning scores (PEWS), most of which are physiology based systems that either produce a numerical score associated with the risk of a patient deteriorating or track a patient's physiological condition and trigger the need for intervention at some threshold. ${ }^{2}$ A primary focus in the development of PEWS has been the establishment of reference ranges for physiological parameters such as heart rate, respiratory rate and systolic blood pressure alongside other factors that may contribute to an algorithm that will allow medical and nursing staff to identify abnormal symptoms before the child becomes critically ill. As Fleming et $a l^{3}$ noted in 2011, the reference ranges at that time were not evidence-based. This is reflected in the assessment of the validity of different PEWS scores in the emergency department in which Sieger et al observed that there was substantial variability in the parameters used and the ranges given for specific physiological measurements. ${ }^{2}$ A survey of National Health Service Trusts in Great Britain carried out between July 2011 and early 2013 determined that $85 \%$ of hospitals providing paediatric inpatient care had implemented a PEWS compared with $25 \%$ in 2005. This was despite, the authors note, the variety of PEWS employed (with only a third based on published systems, the rest from unpublished systems implemented in

Correspondence to Dr Roger C Parslow, Leeds Institute of Cardiovascular and Metabolic Medicine, School of Medicine, Room 8.49, Worsley Building, Clarendon Way, University of Leeds, Leeds, LS2 9JT, UK; r.c.parslow@leeds.ac.uk another hospital or purposely designed for their unit) and a lack of evidence of their effectiveness. ${ }^{4}$ It is notable that the authors of this survey found that respiratory rate and heart rate were included in $88 \%$ of the PEWS used in these hospitals, indicating the perceived value of these two parameters in assessing the sick child. Clearly, it is important that there is good empirical evidence of what are normal heart and respiratory rate ranges in infants and children to set PEWS thresholds.

O'Leary et $a l^{5}$ have addressed the development of age-specific normal ranges for these two common physiological parameters used in PEWS based on routinely collected data at the emergency department of a large children's hospital in Sydney, Australia. They have compared their findings with two studies, one that derived centile charts from data reported in 69 published studies from a mixture of sources including home, community clinical and research settings ${ }^{3}$ and the other that used inpatient data from two tertiary hospitals, ${ }^{6}$ and also against established guidelines for advanced paediatric life support. Their findings indicate that there is still uncertainty over the definitions of normal ranges for heart and respiratory rates in children whether identified in the literature or in current clinical guidelines. Although they have found some agreement with published findings, especially in relation to heart rate, they have identified marked clinical differences in respiratory rate centiles between their data and that of Fleming et al, with their 50th centile coming close to Fleming's 1st in infants but also appearing to be below the advanced paediatric life support minimum range up to 5 years of age. This highlights the dilemma facing clinicians in determining what normal physiology is, and how they should react if the readings fall outside predefined limits. The plethora of nonvalidated PEWS scores in use only serves to exacerbate this dilemma.

How the reference data are determined is all-important: if the reference ranges used in guidance documents are not based on empirical evidence (ie, instead depend on expert opinion) or have been derived from a different setting (perhaps only hospital inpatients) it becomes difficult to see how they can be applied with any degree of confidence in a different setting. O'Leary et al have focused their attention on children attending an emergency department, regarding this setting as the most appropriate as this is an environment in which clinicians' assessment of what is normal physiology in infants and children is critical to their ongoing care.

There has been an increase in the prevalence of obesity in children in recent years and this may have an effect on observations in children under 3 years where body mass is positively related to respiratory rate. Information about body mass was not examined by O'Leary et al and was most probably not available. They have also not explored gender differences or potential differences attributable to ethnic origin. These are considerations that have some merit but the production of age and sex and ethnic origin-specific centile charts would be overcomplicated. It is vital to ensure that clinical decision-making is based on sound evidence but not evidence that is difficult to process in a pressurised clinical environment. The final decision about how sick children are treated is based on clinical judgement in which multiple factors are assessed, many of which are based on established guidelines or reference ranges but some that will include an intuitive response.

We now have more empirical evidence about what is normal in terms of heart and respiratory rates in children presenting at emergency departments. This can be combined with previous published data to provide a more robust basis on which to calculate clinically relevant boundaries but we still lack very large-scale population-based measurements of physiological parameters taken using a standardised protocol. O'Leary et al quite rightly suggest a further meta-analysis or prospective study would aid clinicians in dealing with children from defined subgroups. Perhaps with the expansion of clinical information systems in hospitals and primary care, the opportunity to do this with minimal cost will become increasingly possible using modern data capture and linkage methodology. It would require careful coordination but the result could obviate the need for aggregating data from further single centre studies with differing study protocols.

In an attempt to rationalise the use of PEWS a forthcoming National Institute for Health Research project (PUMA-http:// www.nets.nihr.ac.uk/projects/hsdr/ 


\section{Editorials}

1217817) aims to identify the evidence base for the core components of an effective PEWS and develop an implementation package to enable the use of PEWS in a standardised way across different hospitals. As part of the project the investigators will be developing a 'track and trigger' PEWS tool based on the literature and expert opinion: O'Leary et al's work will provide valuable new evidence on range boundaries for two key physiological parameters that will undoubtedly be included in this tool.

Contributors RCP was commissioned to write this editorial. He wrote and reviewed the text and is solely responsible for its content.

Competing interests None declared.

Provenance and peer review Commissioned; internally peer reviewed.
To cite Parslow RC. Arch Dis Child 2015;100:719720.

Received 13 February 2015

Revised 5 March 2015

Accepted 24 March 2015

Published Online First 20 April 2015

\section{(S) Linked}

http://dx.doi.org/10.1136/archdischild-2014-307401

Arch Dis Child 2015;100:719-720.

doi:10.1136/archdischild-2014-307863

\section{REFERENCES}

1 Pearson GA. ed. Why children die: a pilot study 2006; England (South West, North East and West Midlands), Wales and Northern Ireland. London: CEMACH, 2008:65.
2 Seiger N, Maconochie I, Oostenbrink R, et al. Validity of different pediatric early warning scores in the emergency department. Pediatrics 2013;132: e841-50.

3 Fleming S, Thompson M, Stevens R, et al. Normal ranges of heart rate and respiratory rate in children from birth to 18 years of age: a systematic review of observational studies. Lancet 2011;377: 1011-18.

4 Roland D, Oliver A, Edwards ED, et al. Use of paediatric early warning systems in Great Britain: has there been a change of practice in the last 7 years? Arch Dis Child 2014;99:26-9.

5 O'Leary F, Hayen A, Lockie F, et al. Defining normal ranges and centiles for heart and respiratory rates in infants and children: a cross-sectional study of patients attending an Australian tertiary hospital paediatric emergency department. Arch Dis Child 2015;100: 733-7.

6 Bonafide CP, Brady PW, Keren R, et al. Development of heart and respiratory rate percentile curves for hospitalized children. Pediatrics 2013;131: e1150-7. 